\title{
On The Uniqueness Problems of Entire Functions Concerning Differential Polynomials
}

\author{
Nintu Mandal \\ Chandernagore College, Chandernagore, Hooghly-712136, West Bengal, India
}

\begin{abstract}
We discuss the uniqueness of entire functions concerning differential polynomials. The results extend and generalise recent results of Jiang-Tao Li and Ping Li.
\end{abstract}

Keywords:Entire functions, Uniqueness, Differential Polynomials, Deficient value, Weighted sharing.

\section{Introduction}

Let $f$ and $g$ be two non-constant meromorphic functions defined in the open complex plane $C$. For $a \in C \cup\{\infty\}$ we say that $f$ and $g$ share the value $a \mathrm{CM}$ (counting multiplicities) if the $a$-points of $f$ and $g$ coincide in locations and multiplicities. If we do not consider the multiplicities, we say that $f$ and $g$ share the value aIM(ignoring multiplicities). For standard definitions and notations of the value distribution theory we refer to [1].

We denote by $S(r, f)$ any quantity satisfying $S(r, f)=o\left\{T\left(r_{x} f\right)\right\}$ as $r \rightarrow \infty$ possibly outside a set of finite linear measure. A meromorphic function $a=a(z)$ is called small function of $f$ if $T(r, a)=S(r, f)$.

In [2] and [3] the idea of weighted sharing is introduced which measures how close a shared value is to being shared IM or to being shared CM. We now explain the idea of weighted sharing of values.

Definition 1.1. [2, 3]Let $k$ be a nonnegative integer or infinity. For $a \in C$ we denote by $E_{k}(a, f)$ the set of all $a$ points of $f$ where an $a$-point of multiplicity $m$ is counted $m$ times if $m \leq k$ and $k+1$ times if $m \geq k$. If $E_{k}(a, f)=E_{k}(a, g)$ we say that $f, g$ share the value $a$ with weight $k$.

The definition implies that if $f, g$ share a value $a$ with weight $k$ then $z_{0}$ is a zeroof $f-a$ with multiplicity $m(\leq k)$ if and only if it is a zero of $g-a$ with multiplicity $m(\leq k)$ and $z_{0}$ is a zero of $f-a$ with multiplicity $m(>k)$ if and only if it is a zero of $g-a$ with multiplicity $n(>k)$, where $m$ is not necessarily equal to $n$.

We write $f, g$ share $(a, k)$ to mean that $f, g$ share the value $a$ with weight $k$. Clearly if $f, g$ share $(a, k)$ then $f, g$ share $(a, p)$ for all integers $p, 0 \leq p<k$. Also we note that $f \circ g$ share a value $a$ IM or CM if and only if $f \circ g$ share $(a, 0)$ or $(a, \infty)$ respectively.

Definition 1.2. Let $f$ be a non-constant meromorphic function and $a \in C \cup\{\infty\}$. Fora positive integer $k$ we denote by $N_{k)}(r, a ; f)\left(N_{(k}(r, a ; f)\right)$ the counting function of those $a$-points of $f$ whose multiplicities are less than or equal to $k$ (greater than or equal to $k$ ), where an $a$-point is counted according to its multiplicity.

Also by $\bar{N}_{k)}(r, a ; f)$ and $\left.\bar{N}_{(k}(r, a ; f)\right)$ we denote the corresponding reduced counting functions.

We put

$\lambda(f)=\limsup _{r \rightarrow \infty=\infty} \frac{\log ^{+} T(r, f)}{\log r}$

and

$\delta\left(a_{s} f\right)=1-\limsup _{\gamma \rightarrow \infty} \frac{N\left(r_{s}, a_{p} f\right)}{T(r, f)}$

$\lambda(f)$ and $\delta(a, f)$ are called the order of $f(z)$ and the deficiency of $a$ with respect to $f(z)$ respectively.

Definition 1.3. Let $f$ and $g$ be two non-constant meromorphic functions for anypositive integer $k$ we denote by $N_{(k}^{L}(r, 1 ; f)$ the counting function of zeros of $f-1$ and $g-1$ whose multiplicities are not less than $k$ and about which $f-1$ has larger multiplicity than $g-1$. By $N_{(k}^{E}(r, 1 ; f)\left(N_{k)}^{E}(r, 1 ; f)\right)$ we denote the counting function of zeros of $f-1$ and $g-1$ whose multiplicities are not less (greater) than $k$ and about which $f-1$ and $g-1$ have equal multiplicities. $\operatorname{By}_{(k}^{L}\left(r_{v} 1_{s} f\right), \bar{N}_{(k}^{E}\left(r, 1_{s} f\right)$ and $\bar{N}_{k)}^{E}\left(r, 1_{s} f\right)$ wedenote the corresponding reduced counting functions.

Definition 1.4. Let $f$ be a non-constant meromorphic function we denote by $N_{0}\left(r, 0 ; f^{V}\right)$ the counting function of zeros of $f^{t}$ which are not zeros of $f(f-1)$.

In 1976 C. C. Yang [7] asked: If two transcendental entire functions $f$ and gassume the same zeros with the same multiplicities and that their first derivatives assume the same 1-points with the same multiplicities then what can be said about therelationship between $f$ and $g$ ?

To solve the above question in $1981 \mathrm{~K}$. Shibazaki [6] proved the following:

Theorem A. [6] Let $f$ and $g$ be two entire functions of finite order. If $f^{t}$ and $g^{t}$ share the value $1 \mathrm{CM}$ with $\delta(0, f)>0$ and 0 being lacunary for $g$ then either $f \equiv g$ or $f^{\prime} g^{t} \equiv 1$.

In 1990 H. X. Yi [10] proved the following theorem: 


\section{International Journal of Science and Research (IJSR) \\ ISSN (Online): 2319-7064}

Index Copernicus Value (2013): 6.14 | Impact Factor (2014): 5.611

Theorem B. [10] Let $f$ and $g$ be two non-constant entire functions and let $k$ be a non-negative integer.If $f$ and $g$ share the value $0 \mathrm{CM}, f^{(k)}$ and $g^{(k)}$ share the value $1 \mathrm{CM}$ and $\delta(0, f)>\frac{1}{2}$, then either $f \equiv g$ or $f^{(k)} g^{(k)} \equiv 1$.

Let $f$ be a non-constant meromorphic function. We denote by $P(f)=\sum_{i=0}^{k} a_{i} f^{(i)}$ the differential polynomial of $f$, where $a_{\mathrm{i},}, i=0,1,2, \ldots \ldots, k$ are finite complex numbers and $k$ is a positive integer.

Recently Jiang-Tao Li and Ping Li [4] proved the following theorem:

Theorem C. [4] Let $f$ and $g$ be two non-constant entire functions. Suppose that $f$ and $g$ share the value $0 \mathrm{CM}, P(f)$ and $P(g)$ share the value $1 \mathrm{CM}$ and $\delta(0, f)>\frac{1}{2}$. If $\lambda(f) \neq 1$, then $f \equiv g$ unless $P(f) P(g) \equiv 1$.

Theorem D. [4]. Let $f$ and $g$ be two non-constant entire functions. Suppose that $f$ and $g$ share the value $0 \mathrm{CM}, P(f)$ and $P(g)$ share the value 1 IMand $\delta(0, f)>\frac{4}{5}$. If $\lambda(f) \neq 1$, then $f \equiv g$ unless $P(f) P(g) \equiv 1$.

In this paper we prove the following theorem which extend and generalise Theorem $\mathrm{C}$ and Theorem $\mathrm{D}$.

Theorem 1.1. Let $f$ and $g$ be two non-constant entire functions. Suppose that $f$ and $g$ share the value $0 \mathrm{CM}, P(f)$ and $P(g)$ share $(1, l)$, where $l=0,1,2, \ldots \ldots, \infty$. If $\lambda(f) \neq 1$, then $f \equiv g$ unless $P(f) P(g) \equiv 1$ if one of the following holds:

(i) $l=0$ and $\delta(0, f)>\frac{4}{5}$

(ii) $0<l<\infty$ and $\delta(0, f)>\frac{l+1}{2 l+1}$

(iii) $l=\infty$ and $\delta(0, f)>\frac{1}{2}$

\section{Lemmas}

Lemma 2.1. [5] Let $f$ be a non-constant meromorphic function and let $k$ be a nonnegative integer. Then $T(r, P(f)) \leq T(r, f)+k \bar{N}(r, \infty ; f)$ $+S(r, f)(2.1)$

Lemma 2.2. [4] Suppose that $f(z)$ is a non-constant meromorphic function in the complex plane and $a(z)$ is a small function of $f(z)$. If $f(z)$ is not a polynomial, then

$N(r, 0 ; P(f)-P(a)) \leq T\left(r_{s} P(f)\right)-T(r, f)+$

$N(r, a ; f)+S(r, f)$

(2.2)

and

$N(r, 0 ; P(f)-P(a)) \leq N(r, a ; f)$

$+k \bar{N}(r, \infty ; f)+S(r, f)(2.3)$

Lemma 2.3. [4] Let $f$ and $g$ be two non-constant meromorphic functions such that $f$ and $g$ share the value 1 IM. Let $h=\frac{f^{\prime \prime}}{f^{\prime}}-\frac{2 f^{\prime}}{f-1}-\frac{g^{\prime \prime}}{g^{\prime}}+\frac{2 g^{\prime}}{g-1}$

If $h \neq 0$, then

$T(r ; f) \leq N(r, 0 ; f)+2 \bar{N}(r, \infty ; f)+2 \bar{N}_{(1)}^{L}(r, 1 ; f)+$

$N(r, 0 ; g)+2 \bar{N}(r, \infty ; g)+\bar{N}_{(1}^{L}(r, 1 ; g)+S(r, f)+S(r, g)$.

Lemma 2.4. Let $f$ and $g$ be two non-constant meromorphic functions such that $f$ and $g$ share $\left(1_{x} l\right)$ where $l$ is a positive integer. Let

$h=\frac{f v}{f v}-\frac{2 f s}{f-1}-\frac{g s v}{g s}+\frac{2 g s}{g-1}(2.4)$

If $h \neq 0$, then

$T(r, f) \leq N(r, 0 ; f)+2 \bar{N}(r, \infty ; f)$

$+\bar{N}_{(\mathbb{L}+1}^{L}(r, 1 ; f)+N(r, 0 ; g)$

$+2 \bar{N}(r, \infty ; g)+\bar{N}_{(\mathbb{R}+1}^{L}(r, 1 ; g)$

$+S(r, f)+S(r, g)(2.5)$

Proof. We get from Nevanlinna's second fundamental theorem

$T(r, f)+T(r, g) \leq \bar{N}(r, 0 ; f)+\bar{N}(r, 1 ; f)$

$+\bar{N}(r, \infty ; f)-N_{0}\left(r, 0 ; f^{l}\right)$

$+\bar{N}(r, 0 ; g)+\bar{N}(r, 1 ; g)$

$+\bar{N}(r, \infty ; g)-N_{0}\left(r, 0 ; g^{*}\right)$

$+S(r, f)+S(r, g) \times(2.6)$

Since $f$ and $g$ share $(1, l)$ we get

$$
\begin{aligned}
& \bar{N}(r, 1 ; f)=N_{1}^{E}(r, 1 ; f)+\bar{N}_{(\mathbb{1}+1}^{L}(r, 1 ; f) \\
& +\bar{N}_{\mathbb{R}+1}^{L}\left(r, 1^{*} g\right)+\bar{N}_{(\mathbb{R}+1}^{E}\left(r, 1_{s}, f\right) \\
& +S(r, f)+S(r, g) \\
& =\bar{N}(r, 1 ; g)+S(r, f)+S(r, g) \text {. }
\end{aligned}
$$

Hence

$$
\begin{aligned}
& \bar{N}\left(r_{v}, 1 ; f\right)+\bar{N}\left(r_{v} 1_{s} g\right) \\
& =\bar{N}_{1\}}(r, 1 ; f)+\bar{N}_{(22}(r, 1 ; f)+\bar{N}(r, 1 ; g) \\
& +S(r, f)+S(r, g) \\
& \leq \bar{N}_{1)}\left(r, 1^{*} f\right)+\bar{N}_{(2}\left(r, 1^{*}, g\right)+\bar{N}(r, 1 ; g) \\
& +S(r, f)+S(r, g) \\
& \leq \bar{N}_{1,}(r, 1 ; f)+N(r, 1 ; g)+S(r, f)+S(r, g) \\
& \leq \bar{N}_{1\rangle}(r ; 1 ; f)+T(r, g) \\
& +S(r, f)+S(r, g)
\end{aligned}
$$

From (2.6) and (2.7) we get

$$
\begin{aligned}
T(r, f)+T(r, g) & \leq \bar{N}(r, 0 ; f)+\bar{N}(r, 1 ; f) \\
& +\bar{N}(r, \infty ; f)-N_{0}\left(r, 0 ; f^{v}\right) \\
& +\bar{N}(r, 0 ; g)+\bar{N}(r, 1 ; g) \\
& +\bar{N}(r, \infty ; g)-N_{0}\left(r ; 0 ; g^{\prime}\right) \\
& +S(r, f)+S(r, g) \\
& \leq \bar{N}(r, 0 ; f)+\bar{N}(r ;, \infty f) \\
& -N_{0}\left(r, 0 ; f^{v}\right)+\bar{N}(r, 0 ; g) \\
& +\bar{N}(r, \infty ; g)-N_{0}\left(r, 0 ; g^{v}\right) \\
& +\bar{N}_{1 j}(r, 1 ; f)+T(r, g) \\
& +S(r, f)+S(r, g)
\end{aligned}
$$

Therefore

$T\left(r_{v} f\right) \leq \bar{N}(r, 0 ; f)+\bar{N}\left(r_{v}, \infty ; f\right)-N_{0}\left(r_{v}, 0 ; f^{\prime}\right)$

$+\bar{N}(r, 0 ; g)+\bar{N}(r, \infty ; g)-N_{0}\left(r, 0 ; g^{\prime}\right)$ 


\section{International Journal of Science and Research (IJSR) \\ ISSN (Online): 2319-7064}

Index Copernicus Value (2013): 6.14 | Impact Factor (2014): 5.611

$$
+\bar{N}_{1\}}(r, 1 ; f)+S(r, f)+S(r, g)(2.8)
$$

From (2.4) it can be easily calculated that the possible poles of $h$ occur at (i) multiple zeros of $f$ and $g$, (ii) those 1 points of $f$ and $g$ whose multiplicities are different (iii) those poles of $f$ and $g$ whose multiplicities are different, (iv) zeros $f^{\prime}\left(g^{\prime}\right)$ which arenot the zeros of $f(f-1)(g(g-1))$.

Since $h$ has only simple poles, we have

$$
\begin{aligned}
& N(r, \infty ; h) \leq \bar{N}(r, \infty ; f)+\bar{N}(r, \infty ; g) \\
& +\bar{N}_{(\mathbb{R}+1}^{L}(r, 1 ; f)+\bar{N}_{\mathbb{l}+1}^{L}(r ; 1 ; g) \\
& +\vec{N}_{(2,}(r, 0 ; f)+\bar{N}_{(2)}(r ; 0 ; g) \\
& +N_{0}\left(r, 0 ; f^{\prime}\right)+N_{0}\left(r, 0 ; g^{\prime \prime}\right) \\
& +S(r, f)+S(r, g) \times(2.9)
\end{aligned}
$$

Let $z_{0}$ be a common zero of $f-1$ and $g-1$ then by (2.4), we have $z_{0}$ is a zero of $h$.

Also from (2.4), we have

$m(r, h)=S(r, f)+S(r, g)$.

Hence by (2.9) we have

$$
\begin{aligned}
& \bar{N}_{1)}(r, 1 ; f) \leq N(r, 0 ; h) \leq T(r, h)+o(1) \\
& \leq N(r, \infty ; h)+S(r, f)+S(r, g) \\
& \leq \bar{N}(r, \infty ; f)+\bar{N}(r ; \infty ; g) \\
& +\bar{N}_{(1+1}^{L}(r, 1 ; f)+\bar{N}_{(l+1}^{L}(r ; 1 ; g) \\
& +\mathbb{N}_{(2)}(r, 0 ; f)+\bar{N}_{(2}(r, 0 ; g) \\
& +N_{0}\left(r ; 0 ; f^{\prime}\right)+N_{0}\left(r, 0 ; g^{\prime}\right) \\
& +S(r, f)+S(r, g) \cdot(2.10)
\end{aligned}
$$

From (2.8) and (2.10) we have

$$
\begin{aligned}
& T(r, f) \leq \bar{N}(r, 0 ; f)+\bar{N}(r, \infty ; f)-N_{0}\left(r, 0 ; f^{\prime \prime}\right. \\
& +\bar{N}(r, 0 ; g)+\bar{N}(r, \infty ; g)-N_{0}\left(r, 0 ; g^{\prime}\right) \\
& +\bar{N}(r, \infty ; f)+\bar{N}(r, \infty ; g)+\bar{N}_{(\mathbb{1}+1}^{L}(r, 1 ; f) \\
& +\bar{N}_{(l+1}^{L}(r, 1 ; g)+\bar{N}_{(2}(r, 0 ; f) \\
& +\vec{N}_{(2}(r ; 0 ; g)+N_{0}\left(r, 0 ; f^{\prime}\right)+N_{0}\left(r ; 0 ; g^{\prime}\right) \\
& +S(r, f)+S(r, g) \\
& \leq \bar{N}(r, 0 ; f)+2 \bar{N}(r, \infty ; f)+\bar{N}(r, 0 ; g) \\
& +2 \bar{N}(r, \infty ; g)+\bar{N}_{(1+1}^{L}(r, 1 ; f) \\
& +\bar{N}_{(l+1}^{L}(r, 1 ; g)+\bar{N}_{(2)}(r, 0 ; f) \\
& +\bar{N}_{(22}(r, 0 ; g)+S(r, f)+S(r, g) \\
& \leq N(r, 0 ; f)+2 \bar{N}(r, \infty ; f)+N(r, 0 ; g) \\
& +2 \bar{N}(r, \infty ; g)+\bar{N}_{(l+1}^{L}(r, 1 ; f) \\
& +\bar{N}_{(l+1}^{L}(r, 1 ; g)+S(r, f)+S(r, g)
\end{aligned}
$$

This completes the proof.

Lemma 2.5. [8] Let $f_{j}(j=1,2, \ldots \ldots m+1)$ and $g_{j}(j=1,2, \ldots \ldots m)$ are entire functions satisfying the following conditions:

(i) $\sum_{j=1}^{m} f_{j}(z) e^{g_{j}(z)} \equiv f_{m+1}(z)$;

(ii) The order of $f_{j}(z)$ is less than the order of $e^{g_{k}(z)}$ for $1 \leq j \leq m+1,1 \leq k \leq m$ andthe order of $f_{j}(z)$ is less than the order of $e^{g_{l}(z)-g_{k}(z)}$ for $m \geq 2$ and $1 \leq j \leq m+1,1 \leq l, k \leq m, l \neq k$. Then $f_{j} \equiv 0(j=1,2, \ldots \ldots s m+1)$.
Lemma 2.6. Let $f$ and $g$ be two non-constant entire functions. Suppose that $f$ and $g$ share the value $0 \mathrm{CM}, P(f)$ and $P(g)$ share the value 1IM. If $\lambda(f) \neq 1$ and $P(f) \equiv P(g)$, then $f \equiv g$.

Proof. It can be proved easily with the help of the proof of the Theorem D.

\section{Proof of the Theorem 1.1}

Proof. If $l=0, \infty$ then by Theorem D and Theorem $\mathrm{C}$ we get the result.

Now suppose that $0<l<\infty$. Then we have to consider the following two cases:

Case 1 Any one or both of $P(f)$ and $P(g)$ is constant. Suppose that $P(f)=c$; where $c$ is a finite complex constant.Then

$f \equiv d+\sum_{i=1}^{m} p_{i}(z) e^{\omega_{i} z}$,

whered is a finite complex constant, $p_{\mathrm{i}}(i=1,2, \ldots \ldots m)$ are polynomials and $\alpha_{i}(i=1,2, \ldots \ldots, m)$ are distinct finite complex constants. Since $\quad \lambda(f) \neq 1$ therefore $f \equiv d+\sum_{i=1}^{m} p_{i}(z)$, that is $f$ is apolynomial.Suppose the degree of $f$ is $n$. Then

$N(r, 0 ; f)=n \log r$ and $T(r, f)=n \log r+o(1)$.

Therefore

$\delta(0, f)=1-\underset{r \rightarrow \infty}{\limsup } \frac{N(r, 0 ; f)}{T(r, f)}=0$,

which is a contradiction.

Case $2 P(f), P(g) \neq c$ where $\mathrm{c}$ is a finite complex constant. Let $\quad F=P(f), \quad G=P(g) \quad$ and $H=\frac{F s s}{F^{v}}-\frac{2 F^{r}}{F-1}-\frac{G^{t r}}{G^{y}}+\frac{2 G^{r}}{G-1}$ then $F$ and $G$ share $(1, l)$.

By Milloux's basic result we have

$$
\begin{aligned}
& T(r, f) \leq N(r, 0 ; f)+\bar{N}(r, \infty ; f) \\
& +\bar{N}(r, 1 ; P(f))+S(r, f) \\
& \leq N(r, 0 ; g)+\bar{N}(r, 1 ; P(g))+S(r, f) \\
& \leq T(r, g)+T(r, P(g))+S(r, f) .
\end{aligned}
$$

Hence by Lemma 2.1 we have

$T(r, f) \leq 2 T(r, g)+S(r, f)+S(r, g)$

Similarly we have

$T(r, g) \leq 2 T(r, f)+S(r, f)+S(r, g)$

From (3.1) and (3.2) we have

$S(r, f)=S(r, g)(3.3)$

Now we have to consider the following two subcases:

Subcase1 $H \equiv 0$.

In this case we have

$F=\frac{P G+Q}{R G+S^{x}}(3.4)$

where $P, Q, R$ and $S$ are finite complex constants such that $P S-Q R \neq 0$ :

Now we have to consider the following three cases: 


\section{International Journal of Science and Research (IJSR) \\ ISSN (Online): 2319-7064 \\ Index Copernicus Value (2013): 6.14 | Impact Factor (2014): 5.611}

Case $I P=0$ and $R \neq 0$.

In this case $F=\frac{Q}{R G+S}$

If $S \neq 0$; then

$N\left(r,-\frac{S}{R} ; G\right)=N(r, \infty ; F)$.

By Nevanlinna's Second Fundamental Theorem we have

$T(r, G) \leq N(r, 0 ; G)+N\left(r_{z}-\frac{S}{R} ; G\right)$

$+N(r, \infty ; G)+S(r, G)$

$=N(r, 0 ; G)+N(r, \infty ; F)$

$+N(r, \infty ; G)+S(r, G)$

$=N\left(r_{v}, 0 ; G\right)+S\left(r_{x} G\right)(3.5)$

By Lemma2.2 from (3.5) and (3.3) we have

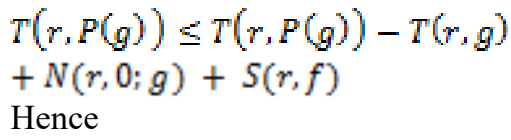

$T(r, g) \leq N(r, 0 ; g)+S(r, f)$

$=N(r, 0 ; f)+S(r, f)(3.6)$

By (3.1) and (3.6) we have

$T(r, f) \leq 2 N(r, 0 ; f)+S(r, f)$.

which contradicts the condition $\delta(0, f)>\frac{\mathbb{1}+1}{21+1^{x}}$

Hence $S=0$ and so $F G=\frac{Q}{R}$. If 1 is a Picard exceptional value of $F$ then $\frac{Q}{R}=1$ otherwise it contradicts the Deficiency Theorem [9]. So $F G \equiv 1$.

If 1 is not a Picard exceptional value of $F$, then there exist a complex number $z_{0}$ such that $F\left(z_{0}\right)=G\left(z_{0}\right)=1$. So $\frac{Q}{R}=1$.

Hence $F G \equiv 1$.

Case IIP $\neq 0$ and $R=0$

In this case we have $F=\frac{P}{5} G+\frac{Q}{5}$.

If $Q \neq 0$, then

$N\left(r, \frac{Q}{S} ; F\right)=N(r, 0: G)$.

Hence by Nevanlinna's Second Fundamental Theorem we have

$T\left(r_{s} F\right) \leq N\left(r_{s} 0 ; F\right)+N\left(r, \frac{Q}{S} ; F\right)$

$+N(r, \infty ; F)+S(r, F)$

$=N(r, 0 ; F)+N(r, 0 ; G)+S(r, F)(3.7)$

By Lemma 2.2 from (3.7) and (3.3) we have

$T\left(r_{v} P(f)\right) \leq T\left(r_{v} P(f)\right)-T\left(r_{v} f\right)+N(r, 0 ; f)$

$+N(r, 0 ; g)+S(r, f)$

Hence

$T(r, f) \leq N(r, 0 ; f)+N(r, 0 ; g)+S(r, f)$

$=2 N(r, 0 ; f)+S(r, f)$.

which contradicts the condition $\delta(0, f)>\frac{\mathbb{1}+1}{21+1^{x}}$

Hence $Q=0$ and so $F=\frac{P}{s} G$.

If 1 is a Picard exceptional value of $F$ then $\frac{P}{5}=1$ otherwise it contradicts the Deficiency Theorem [9]. So $F \equiv G$.
If 1 is not a Picard exceptional value of $F$, then there exist a complex number $z_{0}$ such that $F\left(z_{0}\right)=G\left(z_{0}\right)=1$.

Hence $F \equiv G$.

By Lemma 2.6 we have $f \equiv g$.

Case IIIPR $\neq 0$

From (3.4) it is clear that $\frac{F}{R}$ is a Picard exceptional value of $F$. Hence by Nevanlinna's Second Fundamental Theorem we have

$$
\begin{aligned}
& T\left(r_{v} F\right) \leq N\left(r_{v}, 0 ; F\right)+N\left(r_{v} \frac{P}{R}, F\right) \\
& +N\left(r_{s}, \infty ; F\right)+S\left(r_{v} F\right) \\
& =N\left(r_{v}, 0 ; F\right)+S(r, F)
\end{aligned}
$$

By Lemma 2.2 and (3.8) we have

$T(r, f) \leq N(r, 0 ; f)+S(r, f)_{s}$ which contradicts the condition $\delta(0, f)>\frac{\mathbb{1}+1}{2 \mathbb{1}+1}$

Subcase $2 H \neq 0$.

By Lemma 2.4 we have

$$
\begin{aligned}
& T\left(r_{v} F\right) \leq N\left(r_{v} 0 ; F\right)+\bar{N}_{(\mathbb{R}+1}^{L}\left(r_{v} 1_{w} F\right) \\
& +N(r, 0 ; G)+\bar{N}_{(\mathbb{N}+1}^{L}(r, 1 ; G) \\
& +S\left(r_{v} F\right)+S\left(r_{z} G\right)
\end{aligned}
$$

$\bar{N}_{\mathbb{R}+1}^{L}(r, 1 ; F)+\bar{N}_{\mathbb{R}+1}^{L}(r, 1 ; G)$

$\leq \frac{1}{l} N\left(r, 0 ; F^{v}\right)$

$\leq \frac{1}{\mathbb{l}} N\left(r_{v}, 0 ; F\right)+\frac{1}{\mathbb{l}} \bar{N}\left(r_{x}, \infty_{;} F\right)+S\left(r_{v} F\right)$

From (3.9) and (3.10) we have

$T(r, F) \leq \frac{l+1}{l} N(r, 0 ; F)+N(r, 0 ; G)$

$+S\left(r_{v} F\right)+S\left(r_{v} G\right)(3.11)$

Hence by Lemma 2.2 and (3.11) we have

$T\left(r_{v} P(f)\right) \leq T\left(r_{v} P(f)\right)-T\left(r_{v} f\right)+N\left(r_{v}, 0 ; f\right)$ $+\frac{1}{l} N(r, 0 ; f)+N(r, 0 ; g)+S(r, f)+S(r, g)$

Therefore $T(r, f) \leq N(r, 0 ; f)+\frac{1}{l} N(r, 0 ; f)+N(r, 0 ; g)$ $+S(r, f)+S(r, g)$ $\leq \frac{2 l+1}{l} N(r, 0: f)+S(r, f)$, which contradicts the condition $\delta(0, f)>\frac{\mathbb{1}+1}{2 \mathbb{1}+1^{x}}$ This completes the proof

\section{Acknowledgment}

The Authorwould like to thank the UGC (ERO), India for financial support vide UGC MRP F No. PSW- 105/14-15 (ERO) dated. $26^{\text {th }}$ March, 2015.

\section{References}

[1] W. K. Hayman, Meromorphic Functions, The Clarendon Press, Oxford (1964). 


\section{International Journal of Science and Research (IJSR) \\ ISSN (Online): 2319-7064}

Index Copernicus Value (2013): 6.14 | Impact Factor (2014): 5.611

[2] I. Lahiri, Weighted sharing and Uniqueness of meromorphic functions, Nagoya Math. J., 161(2001), pp.193-206.

[3] I. Lahiri, Weighted value sharing and uniqueness of meromorphic functions, Complex Var. Theory Appl.,Vol.46(3)(2001), pp.241-253.

[4] J-T. Li and P. Li, uniqueness of entire functions concerning differential polynomials, Commun. Korean Math. Soc.,30(2015), No. 2, pp.93-101.

[5] H. Milloux, Les fonctionsmeromorphes et leursderivees, Hermann et Cie., Paris, 1940.

[6] K. Shibazaki, Unicity theorems for entire functions of finite order, Mem. Nat. Defense Acad. (Japan) 21 (1981), no. 3, pp.67-71.

[7] C. C. Yang, On two entire functions which together with their first derivatives have the same zeros, J. Math. Anal. Appl. 56 (1976), no. 1, pp.1-6.

[8] C. C. Yang and H. X. Yi, Uniqueness Theory of Meromorphic Functions, Science Press, Beijing, 1995.

[9] L. Yang, Value Distribution Theory and the New Researches, Science Press, Beijing, 1982.

[10] H. X. Yi, A question of C. C. Yang on the uniqueness of entire functions, Kodai Math. J. 13 (1990), no.1, pp.39-46.

\section{Author Profile}

Dr. Nintu Mandal received the B.Sc., M.Sc. and Ph. D. degrees in Mathematics from University of Kalyani. Dr. Mandal served several Institutions from 2002. Presently Dr. Mandal is an Assistant Professor of Chandernagore College, Government of West Bengal. 